Journal of

Synchrotron

Radiation

ISSN 0909-0495

\section{Advances and synergy of high-pressure sciences at synchrotron sources}

\author{
Haozhe Liu, ${ }^{\text {a* }}$ Tom Duffy, ${ }^{b}$ Lars Ehm, ${ }^{c}$ Wilson Crichton ${ }^{d}$ and Katsutoshi Aoki ${ }^{\mathrm{e}}$

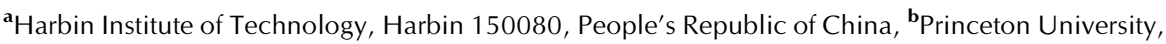 \\ Princeton, NJ 08544, USA, 'National Synchrotron Light Source, Brookhaven National Laboratory, \\ Upton, NY 11973, USA, ${ }^{\mathbf{d} E S R F, ~} 6$ rue Jules Horowitz, BP 220, 38043 Grenoble, France, and $\mathbf{e}_{\text {JAEA/ }}$ \\ SPring-8, 1-1-1 Kouto, Sayo, Hyogo 679-5198, Japan. E-mail: haozheliu@hotmail.com
}

High-pressure research in geosciences, materials science and condensed matter physics at synchrotron sources is experiencing growth and development through synergistic efforts around the world. A series of high-pressure science workshops were organized in 2008 to highlight these developments. One of these workshops, on 'Advances in high-pressure science using synchrotron X-rays', was held at the National Synchrotron Light Source (NSLS), Brookhaven National Laboratory, USA, on 4 October 2008. This workshop was organized in honour of Drs Jingzhu Hu and Quanzhong Guo in celebration of their retirement after up to 18 years of dedicated service to the high-pressure community as beamline scientists at X17 of NSLS. Following this celebration of the often unheralded role of the beamline scientist, a special issue of the Journal of Synchrotron Radiation on Advances and Synergy of High-Pressure Sciences at Synchrotron Sources was proposed, and we were pleased to invite contributions from colleagues who participated in the workshop as well as others who are making similar efforts at synchrotron sources worldwide.

In this special issue, several trends highlighting the advances and synergy of high-pressure sciences have been documented in these first 12 papers of the issue:

(i) Development of cutting-edge high-pressure programs at non-dedicated beamlines. This trend is becoming dominant at many synchrotron sources, and is removing the boundary between dedicated and non-dedicated high-pressure beamlines. An excellent example is the 'Achievements in highpressure science at the high-brilliance energy-dispersive X-ray absorption spectrometer of ESRF, ID24', by G. Aquilanti et $a l$., in which the special requirements for fine focusing and high flux at the sample location, accommodation of highpressure equipment, and challenging scientific projects from the user community were all fully considered from the very beginning of the design and upgrade of the beamline optics and end-station. Several other beamlines featuring various advanced X-ray techniques and highlighting synergistic efforts are also represented in this special issue, such as in 'Phonon spectroscopy at high pressure by inelastic X-ray scattering' (at ESRF) by V. M. Giordano et al.; 'Sound velocities of compressed $\mathrm{Fe}_{3} \mathrm{C}$ from simultaneous synchrotron X-ray diffraction and nuclear resonant scattering measurements' (at APS) by L. Gao et al.; 'Development of an energy-domain ${ }^{57} \mathrm{Fe}-\mathrm{Mössbauer}$ spectrometer using synchrotron radiation and its application to ultrahigh-pressure study with a diamond anvil cell' (at SPring-8) by T. Mitsui et al.; and 'X-ray magnetic spectroscopy at high pressure: performance of SPring- 8 BL39XU' by N. Kawamura et al.

One key feature of the trend toward synergistic efforts at beamlines is that the user groups are playing a more important role than ever before. The development of a 'Portable laser heating system for diamond anvil cells' (built at the BGI and used at the ESRF) by L. Dubrovinsky et al. is a good example. The benefits from participation of user groups in the synergistic efforts is not limited to technical input or incorporation of advanced high-pressure techniques at non-dedicated highpressure beamlines, but also serves to broaden scientific research goals. The latter provides the driving force for the major scientific developments that go beyond synchrotron technique development. The synergistic effort for high-pressure sciences at synchrotron sources is mostly in partnership mode, such as the 'High Pressure Synergetic Consortium' (HPSynC) at the APS, Argonne, which was founded in 2007, as well as the proposed 'Partnership for Extreme Condition Science' (PECS) at the ESRF and ILL, Grenoble, initiated to encompass the whole European high-pressure science community. We believe that excellent and exciting high-pressure research from non-dedicated facilities will join the effort in the near future.

(ii) Further development and integration at dedicated highpressure beamlines. The traditional dedicated high-pressure beamlines at synchrotron facilities continue to take the leading role in cutting-edge technical development. For example, the stress and rheology research under high-pressure and high-temperature conditions are of strong interest to the Earth science community. The following papers in this issue demonstrate the effort and progress: 'A combination of a Drickamer anvil apparatus and monochromatic X-rays for stress and strain measurements under high pressure' (at APS) by N. Nishiyama et al.; 'In situ rheological measurements at extreme pressure and temperature using synchrotron X-ray diffraction and radiography' by P. Raterron and S. Merkel; and 'Stress measurement under high pressure using Kawai-type multi-anvil apparatus combined with synchrotron radiation' (at SPring-8) by Y. Nishihara et al.

The integration of multiple techniques at the dedicated high-pressure facilities remains active after the success of HPCAT at APS. One example of this is the paper 'A system for 
measuring elastic wave velocity under high pressure and high temperature using a combination of ultrasonic measurement and the multi-anvil apparatus at SPring-8' by Y. Higo et al.

(iii) Novel techniques for high-pressure research. Many nonbeamline-related developments will push the frontier of highpressure science as well. For example, 'Laser heating in diamond anvil cells: developments in pulsed and continuous techniques' by A. F. Goncharov et al. shows the technical advances and challenges for the high-pressure and hightemperature domain. Last but not least, a very impressive development, 'Combined high pressure and heavy-ion irradiation: a novel approach' by M. Lang et al. highlights a new avenue to high-pressure research for irradiation modification of structures and properties, which will undoubtedly have many geological and engineering applications. New science frontiers will be reached by combination of novel techniques, and the territory of high-pressure research will be pushed to more interdisciplinary areas than ever before.

Finally, we would like to thank the support provided for the workshop by the Consortium for Materials Property Research in Earth Sciences (COMPRES), the Carnegie-DOE Alliance Center (CDAC), Harbin Institute of Technology (HIT) and NSLS. We acknowledge Professor Gene Ice for his kind support and helpful suggestions for this special issue, and the many reviewers who contributed their time and effort to evaluate and improve the submitted manuscripts. 